\title{
A Study on the Stoichiometry of One-Dimensional Nanostructures
}

\author{
Keivan Davami, ${ }^{1,2}$ Mehrdad Shaygan, ${ }^{3}$ Nazli Kheirabi, ${ }^{4}$ and Hessam Ghassemi ${ }^{5}$ \\ ${ }^{1}$ Department of Mechanical Engineering and Applied Mechanics, University of Pennsylvania, Philadelphia, PA 19104, USA \\ ${ }^{2}$ Department of Mechanical Engineering, Widener University, One University Place, Chester, PA 19013, USA \\ ${ }^{3}$ Advanced Microelectronic Center Aachen (AMICA), AMO GmbH, Otto-Blumenthalstraße 25, 52074 Aachen, Germany \\ ${ }^{4}$ Department of Electrical and Computer Engineering, University of Waterloo, Waterloo, ON, Canada N2L 3G1 \\ ${ }^{5}$ Department of Materials Science and Engineering, Drexel University, Philadelphia, PA 19104, USA
}

Correspondence should be addressed to Keivan Davami; kdavami@seas.upenn.edu

Received 19 April 2015; Revised 7 June 2015; Accepted 8 June 2015

Academic Editor: Da-Ren Hang

Copyright (C) 2015 Keivan Davami et al. This is an open access article distributed under the Creative Commons Attribution License, which permits unrestricted use, distribution, and reproduction in any medium, provided the original work is properly cited.

\begin{abstract}
While attributes such as small dimensions, low power consumption, fast sensor response, and a wide range of detection give onedimensional nanostructures excellent potential to revolutionize sensor and detector industries, challenges to achieving uniform stoichiometry pose significant obstacles to their commercial use. Diverse characteristics arise from nanostructures with variable compositions and morphologies. Thus, investigation of physical properties of nanostructures would be pointless if one cannot assure the exact stoichiometry of the material. We studied the stoichiometry of ZnTe nanowires grown via the vapor-liquid-solid method. Different microscopy and composition analysis methods were exploited to study the stoichiometry of the nanowires. It was observed that nonstoichiometric wires had relatively higher defect concentrations. The temperature profile along the substrate during nanowire growth was found to be the reason for the formation of nanowires with different stoichiometries.
\end{abstract}

\section{Introduction}

One-dimensional semiconductor nanostructures such as nanowires (NWs) have been extensively studied recently due to their diversity and inherent features arising from their geometry and confinement effects. They have the potential to be utilized in future advanced nanoelectronic and optoelectronic devices, which can be well implemented by the bottomup methods such as chemical vapor deposition techniques $[1,2]$.

Methodologies to synthesize nanostructures with predefined composition and stoichiometry are highly desired. The literature is replete with the exploration of the optimum growth conditions for one-dimensional nanostructures using various methods such as the vapor-liquid-solid (VLS) method; however, an accurate control of the stoichiometry of the one-dimensional nanostructures has only been the focus of a few reports [3-8].
Stoichiometry of the nanowires affects their performance when they are used in a variety of devices and for different applications. The nonuniformity in the composition of NWs grown with similar growth parameters or even in one batch and on one substrate makes them unsuitable for commercialized device fabrication. Therefore precise control over the stoichiometry of nanostructures is a critical issue. For example, semiconductor ternary alloyed nanowires $[9,10]$ are favorable in applications where band gap tunability is required; however the band gap is greatly sensitive to alloy composition. In thermoelectric applications, the thermopower and electrical conductivity of the nanowires, which determine the thermoelectric efficiency of the device, are highly sensitive to stoichiometry and a very precise control (better than a few percent) is required over the material composition [6]. Stoichiometry also affects the gas sensing response of one-dimensional-based sensors as it affects that of the bulk and thin film-based gas sensors [11]. Kumar et al. [8] 
observed different responses from two one-dimensionalbased gas sensors despite their comparable dimensions and similar test conditions. This declares that besides dimensions there are other influential properties that are vital in the performance of the gas sensors.

Since in the VLS method tuning the growth parameters such as the growth temperature, pressure, and gas flow can be easily conducted, stoichiometric nanowires are achievable. It was previously shown that stoichiometric InSb nanowire growth is possible only in a very limited temperature range via the VLS method [12]. At higher (or lower) temperatures the nanowires became rich in In (or $\mathrm{Sb}$ ) and poor in $\mathrm{Sb}$ (or In). Besides the parameters that directly affect the growth of the nanowires in the VLS process such as the temperature, pressure, gas flow, and type of the catalyst in some cases, adding another element such as water vapors might be used to contribute to achieving the required stoichiometry [8-13].

The II-VI family of semiconductors and their alloys are promising materials in room temperature radiation detection [14], thermoelectric applications [15], and also photonic applications [16]. ZnTe is a direct band gap group II-VI semiconductor with an energy band gap of $2.26 \mathrm{eV}$, a suitable material to be utilized in pure green optoelectronics [16]. Herein, we focus on the growth conditions for stoichiometric $\mathrm{ZnTe}$ nanowire synthesis, as well as observation of structural defects in nonstoichiometric wires.

\section{Experimental Procedure}

The VLS growth and characterization of ZnTe nanowires were reported before $[17,18]$. As a summary of the growth procedure, Si substrates with $1 \mathrm{~nm}$ of thermally evaporated gold were placed in a quartz tube of a two-zone furnace under the downstream heaters. High purity (99.99\% Aldrich) ZnTe powder $(0.1 \mathrm{~g})$ was placed in a quartz boat and positioned $\sim 25 \mathrm{~cm}$ away from the $\mathrm{Si}$ pieces in the tube under the upstream heaters. Under an argon flow inserted from the left side (upstream), both the upstream and downstream heaters were heated at $15^{\circ} \mathrm{C} / \mathrm{min}$. Different combinations of temperatures for the source material and substrate heaters were tested to find the optimized growth conditions for the synthesis of nanowires. The procedure for finding the optimized growth parameters is explained in [18] and a summary is presented here. The source and substrate temperatures were varied in the range of $700^{\circ} \mathrm{C}-850^{\circ} \mathrm{C}$ and $450^{\circ} \mathrm{C}-600^{\circ} \mathrm{C}$, respectively, in $50^{\circ} \mathrm{C}$ steps. A mixture of argon and hydrogen with a ratio of $5: 1$ as the carrier gas was inserted into the furnace with a 30 SCCM flow rate. The pressure inside the quartz tube was maintained at 300 Torr. At the end of each $90 \mathrm{~min}$ of growth time, the $\mathrm{Si}$ substrates covered with the nanowires were taken out after cooling the furnace to room temperature. The optimized growth temperature was determined based on the morphology and composition of the nanowires as described below.

The morphology and stoichiometry of the as-grown nanowires were examined using a scanning electron microscope (SEM) (JEOL JSM-7401F) and a high transmission electron microscope (TEM) (JEOL JEM-2100F) equipped with an energy dispersive X-ray spectrometer (EDX). Also,
X-ray diffraction analysis (XRD) (D/Max-2500/PC, Rigaku Co.) and photoluminescence (PL) spectra (Alpha300 Raman spectrometer, WITec) were used to study the composition and band gap energy of the nanowires, respectively. In order to study the crystal structure of ZnTe nanowires, the XRD spectra of the samples were measured in the scanning angle range of $5^{\circ}-90^{\circ}$ with a scanning speed of $4^{\circ} / \mathrm{min}$. The Joint Committee of Powder Diffraction Standard (JCPDS) card file data was employed to interpret the observed peaks where the standard diffraction patterns of ZnTe (JCPDS card number 15-0746) were assigned as references.

\section{Results and Discussion}

Figures 1(a) and 1(e) show representative SEM pictures of mats of nanowires grown in the same growth temperature and with the same growth parameters but in different runs. The probing spots were both approximately in the middle of the $15 \mathrm{~mm}$ long substrates. The length of the nanowires was in the range of 20 to $100 \mu \mathrm{m}$ and their diameters varied from 20 to $150 \mathrm{~nm}$. Different shapes of the nanowires including tapered nanowires, nanoribbons, and nanosaws could be achieved depending on the growth parameters. It was observed that at a source temperature of $750^{\circ} \mathrm{C}$ and substrate temperature of $500^{\circ} \mathrm{C}$ the nanowires with cylindrical morphologies were the majority of the densely grown nanowires. Panels (b) and (f) depict higher magnification SEM images of individual ZnTe nanowires having various diameters. The EDS analysis of individual nanowires was performed to investigate their composition. The EDS results proved that stoichiometric nanowires with similar compositions were grown in different runs but with the same growth condition (Figures 1(d) and 1(h)). As can be seen in panels (c) and (g), the atomic percentages of $\mathrm{Zn}$ and Te for both individual nanowires are similar. Thus, this combination of temperatures was chosen as the optimized condition. A study on the effect of different growth parameters on the morphology of the nanowires was reported before [18].

PL spectra obtained from a pile of ZnTe nanowires scratched from the substrate are shown in Figure 2(a). The predominant near-band-gap emission is observed at $553 \mathrm{~nm}$ wavelength which corresponds to band gap energy of $2.24 \mathrm{eV}$ (green emission) for the nanowires. Figure 2(b) shows the XRD results of the NWs. As can be seen in this figure, the crystalline structure of our ZnTe NWs was determined as zincblende since three main peaks can be indexed as (111), (220), and (311) peaks in the XRD patterns corresponding to $2 \theta=25.29^{\circ}, 41.08^{\circ}$, and $49.49^{\circ}$, respectively. These results are in good agreement with the main peaks of crystalline ZnTe nanowires reported by Yong et al. [19].

The VLS mechanism has been studied in detail previously $[20,21]$. At first, the thin gold film on the substrates melts and forms gold droplets colloquially called "gold islands." The gold islands can adsorb the source material vapor and form a catalytic liquid alloy phase. When the droplets become supersaturated crystal growth subsequently starts. The growth originates from nucleated seeds at the liquid-solid interface [22]. As previously reported [18], the key element affecting the morphology and stoichiometry of the VLS-grown nanowires 


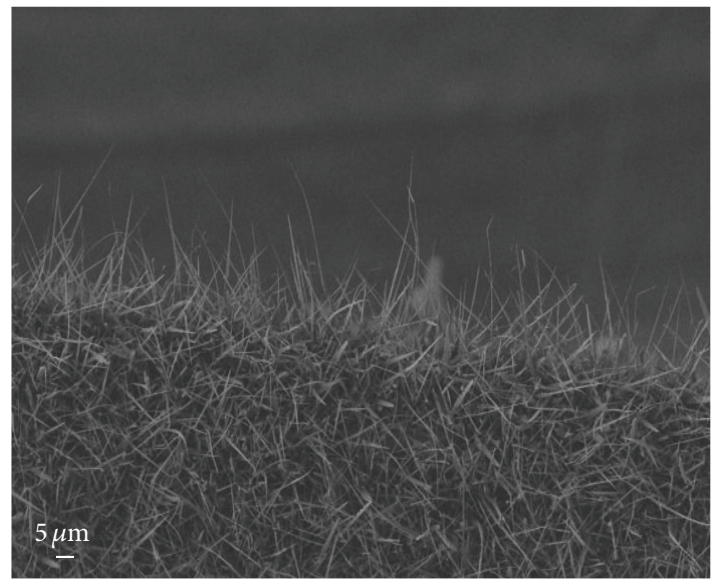

(a)

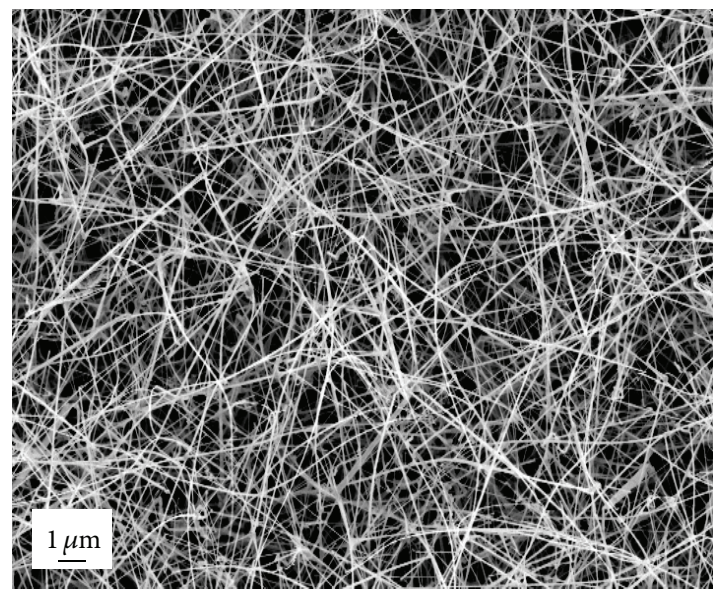

(e)

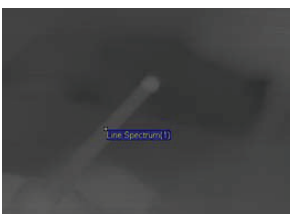

Elem. (Wt.\%)(At.\%)

\begin{tabular}{lll}
\hline $\mathrm{Si} \mathrm{K}$ & 12.71 & 33.90
\end{tabular}

Zn K $\quad 27.43 \quad 31.43$

$\begin{array}{lll}\text { Te L } & 57.61 & 33.81\end{array}$

$\begin{array}{ll}\text { Au M } & 2.25 \quad 0.85\end{array}$

(b)

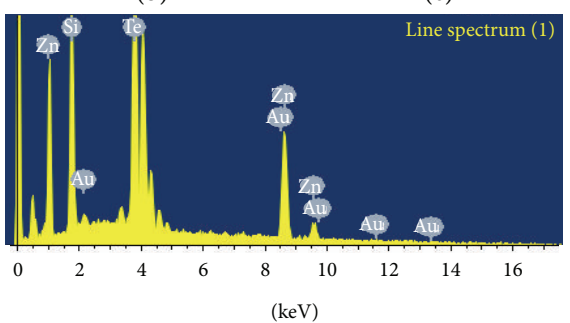

(d)

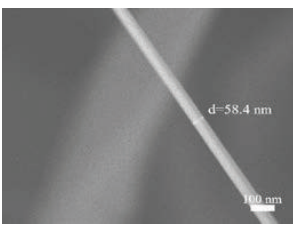

Elem. (Wt.\%)(At.\%)

\begin{tabular}{lll}
\hline Si K & 12.52 & 33.24
\end{tabular}

Zn K $\quad 28.16 \quad 32.11$

$\begin{array}{llll}\text { Te L } & 59.32 & 34.65\end{array}$

$\begin{array}{lll}\mathrm{Au} M & 0.0 & 0.0\end{array}$

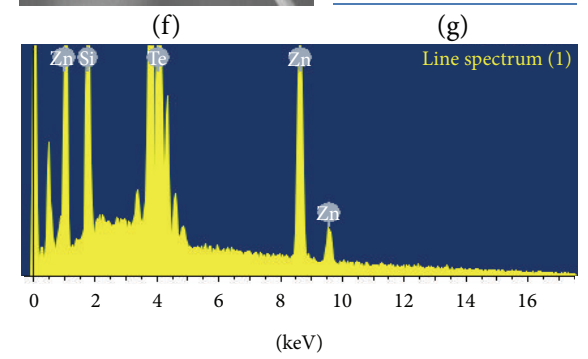

(h)

FIGURE 1: (a) and (e) Representative SEM images of mats of ZnTe nanowires grown in different runs but with the same growth parameters. The source and substrate temperatures were $750^{\circ} \mathrm{C}$ and $500^{\circ} \mathrm{C}$, respectively. (b) and (f) Higher magnification SEM images of single ZnTe nanowires having various diameters. (c) and (g) The elemental distribution of ZnTe nanowires obtained from EDX analysis shown in (d) and (h).

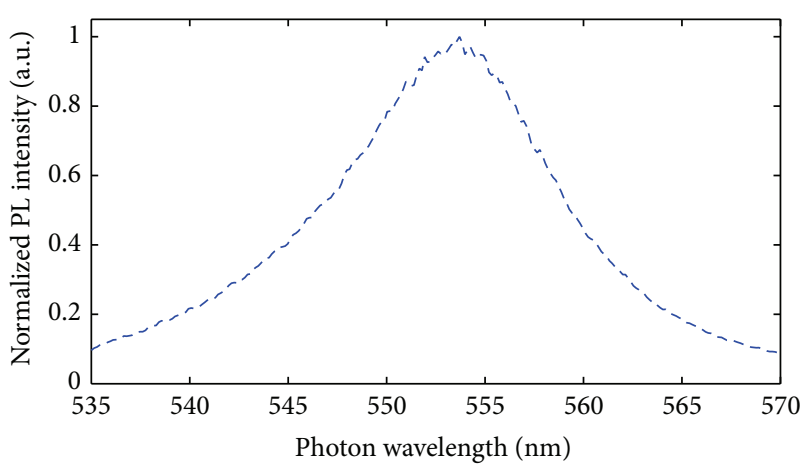

(a)

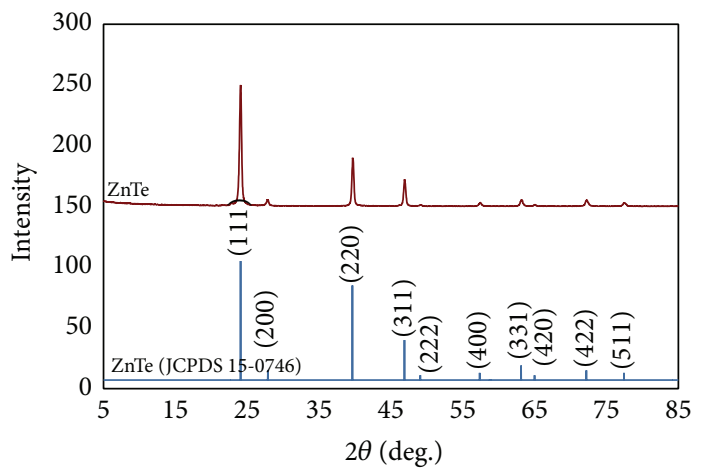

(b)

FIgURE 2: (a) PL spectra of grown ZnTe nanowires. (b) XRD analysis of ZnTe nanowires. 


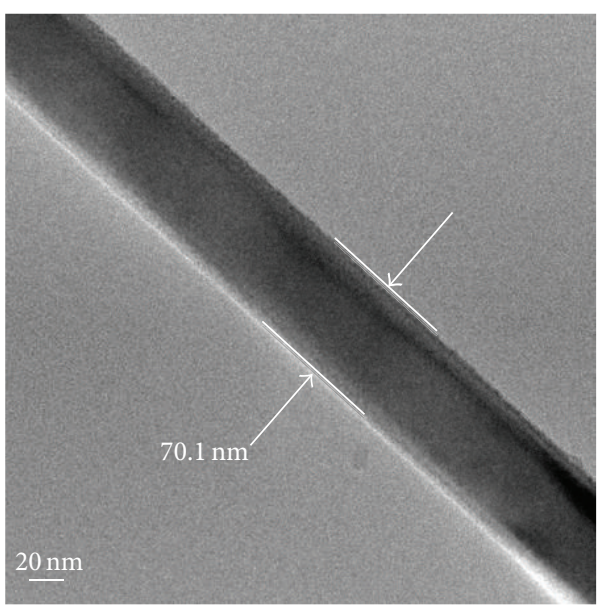

(a)

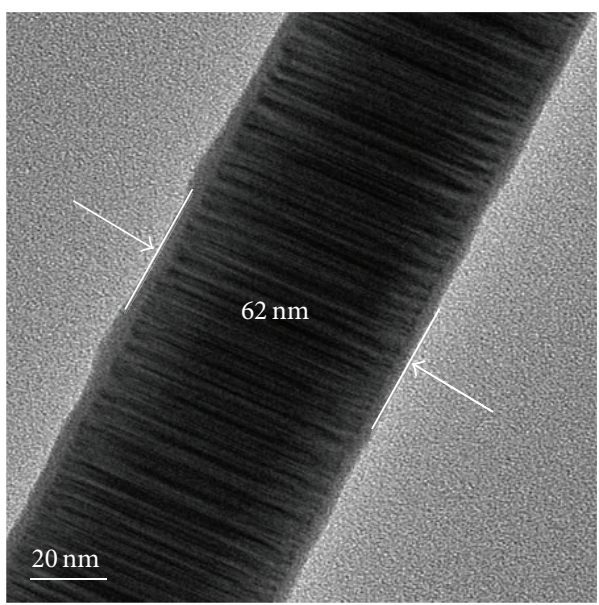

(c)

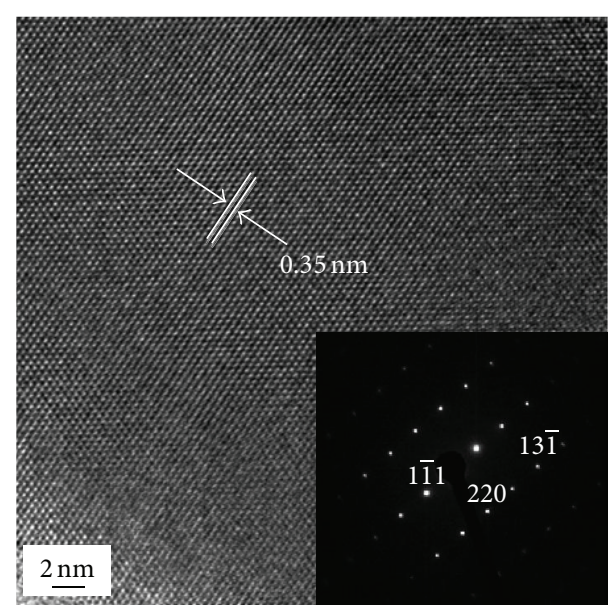

(b)

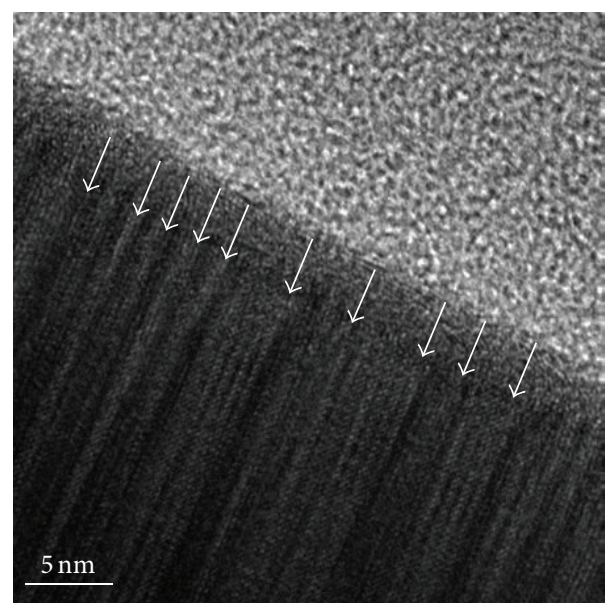

(d)

Figure 3: (a) and (b) Low and high magnification TEM image of ZnTe nanowire grown in the optimized growth temperature (the temperatures of the source and substrate were $750^{\circ} \mathrm{C}$ and $500^{\circ} \mathrm{C}$, resp.). The inset in (b) shows the SAED pattern. (c) and (d) Low and high magnification TEM image of ZnTe nanowire grown at a higher growth temperature (the temperatures of the source and substrate were $850^{\circ} \mathrm{C}$ and $600^{\circ} \mathrm{C}$, resp.) depicting the stacking faults shown by white arrays in panel (d).

is the temperature of the substrate and the source [12]. The optimum substrate and source material temperatures for the growth of stoichiometric ZnTe NWs with a cylindrical shape were $500 \pm 20^{\circ} \mathrm{C}$ and $750 \pm 20^{\circ} \mathrm{C}$, respectively. Temperatures higher or lower than these resulted in the growth of noncylindrical and/or nonstoichiometric nanowires having a relatively higher amount of crystal defects such as stacking faults. When the substrate temperature was reduced to lower than $450^{\circ} \mathrm{C}$, almost no nanowires grew on the substrate. Also, at source temperatures lower than $700^{\circ} \mathrm{C}$ NWs grew with a very low growth density. At temperatures higher than $850^{\circ} \mathrm{C}$, nanoribbons, tapered nanowire, and nanosaws were the majority of the nanostructures instead of nanowires [18].

Besides the composition of the nanowires, growth velocity, $v$, in the VLS process also depends on the synthesis temperature, $T$, and is proportional to $[(\Delta \mu) / k T]^{2}$, where $\Delta \mu$ is a thermodynamic parameter called supersaturation [23]. Supersaturation is defined as the chemical potential difference between adatoms of growth species in the vapor and the solid crystal phases and is the dominant parameter that affects the nucleation and growth rate of the nanowires [24]. Since the nanowires had a wide range of lengths (20 to $100 \mu \mathrm{m}$ ), it was not possible to accurately estimate the growth velocity for $\mathrm{ZnTe}$ nanowires in this study.

In order to study how the stoichiometry affects the nanowire structure, HRTEM was performed on several nanowires grown at the optimized growth temperature for the growth of stoichiometric NWs (the temperatures of the source and substrate were $750^{\circ} \mathrm{C}$ and $500^{\circ} \mathrm{C}$, resp.) and at the nonoptimized growth temperatures (the temperatures of the source and substrate were $850^{\circ} \mathrm{C}$ and $600^{\circ} \mathrm{C}$, resp.). Figures 3(a) and 3(b) show a low and high magnification image of a $71 \mathrm{~nm}$ in diameter individual stoichiometric $\mathrm{ZnTe}$ nanowire, respectively. The EDX analysis of the nanowire was performed inside the TEM (not shown here). The analysis result showed the presence of $\mathrm{Zn}$ and $\mathrm{Te}$ with almost equal atomic percentages, a limited amount of oxygen and silicon, and mostly, at the top of the nanowire, the gold atoms. 
The HRTEM image proves that the ZnTe NW shown here is a single crystal nanostructure (Figures $3(\mathrm{~b})$ and $3(\mathrm{~d})$ ). The single crystallinity of the nanowire can also be realized from the regular spot patterns of the SAED picture (Figure 3(b), inset), where the diffraction spots in the SAED pattern can be indexed to cubic FCC structure. The lattice constant $a$ and lattice spacing were measured to be $\sim 6.1 \AA$ and $\sim 3.5 \AA$, respectively. The lattice spacing corresponds to the $d$-spacing of the (111) plane of the ZnTe FCC structure.

Multiple parallel alternately bright and dark lines observed in Figures 3(c) and 3(d) where the ZnTe nanowire was grown at a temperature higher than the optimized growth temperature confirm the layered structure of the ZnTe nanowire. These striations were found to be stacking faults; these defects together with twinning were reported to be the common planar defects in ZnTe nanowires $[19,25]$.

Growth temperature can strongly affect the crystal structure of nanowires. It was previously reported that lower growth temperatures can greatly reduce the density of stacking faults regardless of the nanowire diameters, while a mixture of wurtzite/zincblende structures and twinning planes could be found in NWs grown at higher growth temperatures [26]. It is worthy to note that stoichiometry variations attributed to temperature are the reason for the common observed defects such as stacking faults and twinning in IIVI group compound semiconductor nanomaterials [27, 28]. Wang et al. [29] reported a certain growth temperature for observing these defects in ZnSe nanowires, where, at lower temperatures, most of the nanowires were defect-free. They explained that coherent twin boundaries might be formed due to the released elastic energy and residual stress at the interface of gold particles and ZnSe nanowires.

In order to investigate the relationship between composition and diameter of nanowires, a detailed study was conducted for a significant number of ZnTe nanowires using EDX analysis of the TEM. The samples were chosen from different spots of the substrate with a length of $15 \mathrm{~mm}$. The optimized growth temperature was selected for the growth of stoichiometric nanowires as described above. It was realized that, even on one single substrate with a length of $\sim 15 \mathrm{~mm}$, nanowires at the two ends of the substrate did not have the same stoichiometry. In general, nanowires grown at the areas of the substrate with a higher temperature had larger diameters and higher percentages of Te (Figure 4). This was attributed to a $\sim 20^{\circ} \mathrm{C}$ temperature difference between the two ends of the substrate due to the temperature profile inside the quartz tube [18]. Figure 4 shows the atomic percentage of $\mathrm{Zn}$ and $\mathrm{Te}$ for nanowires with different diameters. Since the composition is extremely sensitive to the substrate temperature, whenever the temperature gradient exists along the growth tube (which is usually the case), uniformity and consistent stoichiometry of the nanostructures are not achievable on different spots of any reasonably long substrate (more than a few $\mathrm{mm}$ ). An efficient method to grow nanostructures with uniform compositions is to place the substrate vertically in the furnace tube, directly facing the gas flux of precursor atoms to assure a uniform growth temperature at each point of the substrate [14].
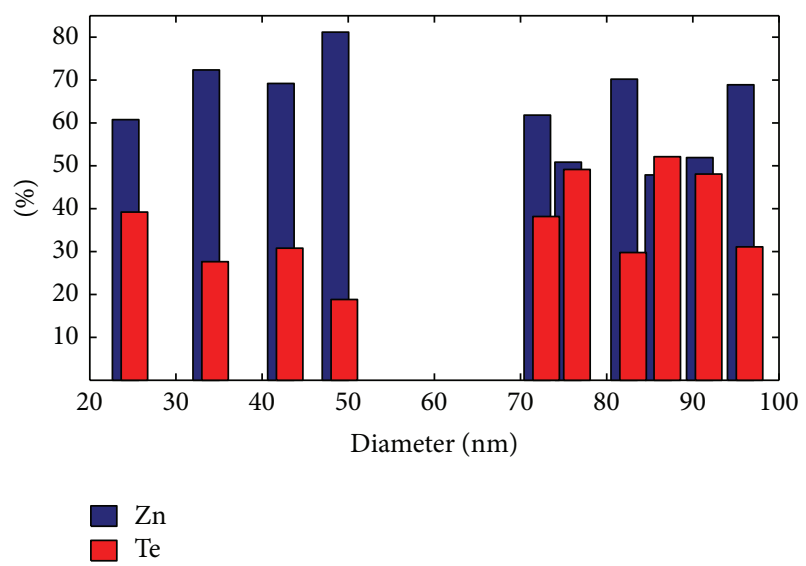

FIgURE 4: Atomic percentage of $\mathrm{Zn}$ and Te versus diameter in the VLS-grown nanowires.

\section{Conclusions}

The stoichiometry as well as defect density significantly affects the properties of nanowires. Fabrication of nanowires with uniform properties is imperative in opening their way to the realm of commercialized devices. Through the VLS method, it is possible to accurately control the stoichiometry of nanowires by adjusting the growth parameters. Herein, it was shown that among different variables in the synthesis of nanowires via the VLS method, source and substrate temperatures play a key role and need to be optimized precisely in order to synthesize stoichiometric nanowires with cylindrical morphology. TEM study of nanowires with different stoichiometries showed a relatively higher concentration of defects in the nonstoichiometric nanowires compared to the stoichiometric nanowires. A solution was presented for achieving a more uniform temperature across the substrate which is vital for the growth of stoichiometric nanowires.

\section{Conflict of Interests}

The authors declare that there is no conflict of interests regarding the publication of this paper.

\section{References}

[1] W. Lu and C. M. Lieber, "Nanoelectronics from the bottom up," Nature Materials, vol. 6, no. 11, pp. 841-850, 2007.

[2] Y. Li, F. Qian, J. Xiang, and C. M. Lieber, "Nanowire electronic and optoelectronic devices," Materials Today, vol. 9, no. 10, pp. $18-27,2006$.

[3] J. Lu, H. Liu, C. Sun et al., "Optical and electrical applications of $\mathrm{ZnS}_{x} \mathrm{Se}_{1-x}$ nanowires-network with uniform and controllable stoichiometry," Nanoscale, vol. 4, no. 3, pp. 976-981, 2012.

[4] L. Junpeng, S. Cheng, Z. Minrui et al., "Facile one-step synthesis of $\mathrm{CdS}_{x} \mathrm{Se}_{1-x}$ nanobelts with uniform and controllable stoichiometry," The Journal of Physical Chemistry C, vol. 115, no. 40, pp. 19538-19545, 2011. 
[5] G. Li, Y. Jiang, Y. Wang et al., "Synthesis of $\mathrm{CdS}_{X} \mathrm{Se}_{1-X}$ nanoribbons with uniform and controllable compositions via sulfurization: optical and electronic properties studies," Journal of Physical Chemistry C, vol. 113, no. 39, pp. 17183-17188, 2009.

[6] N. Peranio, E. Leister, W. Töllner, O. Eibl, and K. Nielsch, "Stoichiometry controlled, single-crystalline $\mathrm{Bi}_{2} \mathrm{Te}_{3}$ nanowires for transport in the basal plane," Advanced Functional Materials, vol. 22, no. 1, pp. 151-156, 2012.

[7] U. Philipose and G. Sapkota, "Defect formation in InSb nanowires and its effect on stoichiometry and carrier transport," Journal of Nanoparticle Research, vol. 15, article 2129, 11 pages, 2013.

[8] M. Kumar, B. R. Mehta, V. N. Singh et al., "The role of stoichiometry of indium and oxygen on gas sensing properties of indium oxide nanostructures," Applied Physics Letters, vol. 96, no. 12, Article ID 123114, 2010.

[9] K. Davami, J. Pohl, M. Shaygan et al., "Bandgap engineering of $\mathrm{Cd}_{x} \mathrm{Zn}_{1-x}$ Te nanowires," Nanoscale, vol. 5, no. 3, pp. 932-935, 2013.

[10] A. Pan, H. Yang, M. Liu, R. Yu, B. Zou, and Z. Wang, "Colortunable photoluminescence of alloyed $\mathrm{CdS}_{x} \mathrm{Se}_{1-x}$ nanobelts," Journal of the American Chemical Society, vol. 127, pp. 1569215693, 2005.

[11] G. Korotcenkov, V. Brinzari, J. R. Stetter, I. Blinov, and V. Blaja, "The nature of processes controlling the kinetics of indium oxide-based thin film gas sensor response," Sensors and Actuators, B: Chemical, vol. 128, no. 1, pp. 51-63, 2007.

[12] U. Philipose, G. Sapkota, J. Salfi, and H. E. Ruda, "Influence of growth temperature on the stoichiometry of InSb nanowires grown by vapor phase transport," Semiconductor Science and Technology, vol. 25, no. 7, Article ID 075004, 2010.

[13] M. Kumar, V. N. Singh, B. R. Mehta, and J. P. Singh, “Tunable synthesis of indium oxide octahedra, nanowires and tubular nanoarrow structures under oxidizing and reducing ambients," Nanotechnology, vol. 20, no. 23, Article ID 235608, 2009.

[14] Y. Eisen and A. Shor, "CdTe and CdZnTe materials for roomtemperature X-ray and gamma ray detectors," Journal of Crystal Growth, vol. 184-185, pp. 1302-1312, 1998.

[15] N. Mingo, "Thermoelectric figure of merit of II-VI semiconductor nanowires," Applied Physics Letters, vol. 85, no. 24, pp. 5986-5988, 2004.

[16] M. Shaygan, M. Meyyappan, and J. S. Lee, "Nanowire field effect transistors in optoelectronics," in Nanowire Field Effect Transistors: Principles and Applications, pp. 187-224, Springer, New York, NY, USA, 2014.

[17] K. Davami, H. M. Ghassemi, R. S. Yassar, J.-S. Lee, and M. Meyyappan, "Thermal breakdown of ZnTe nanowires," ChemPhysChem, vol. 13, no. 1, pp. 347-352, 2012.

[18] K. Davami, D. Kang, J. S. Lee, and M. Meyyappan, "Synthesis of ZnTe nanostructures by vapor-liquid-solid technique," Chemical Physics Letters, vol. 507, p. 208, 2011.

[19] K.-T. Yong, Y. Sahoo, H. Zeng, M. T. Swihart, J. R. Minter, and P. N. Prasad, "Formation of ZnTe nanowires by oriented attachment," Chemistry of Materials, vol. 19, no. 17, pp. 41084110, 2007.

[20] R. S. Wagner and W. C. Ellis, "Vapor-liquid-solid mechanism of single crystal growth," Applied Physics Letters, vol. 4, no. 5, pp. 89-90, 1964.

[21] T. J. Trentler, K. M. Hickman, S. C. Goel, A. M. Viano, P. C. Gibbons, and W. E. Buhro, "Solution-liquid-solid growth of crystalline III-V semiconductors: an analogy to vapor-liquidsolid growth," Science, vol. 270, no. 5243, pp. 1791-1794, 1995.
[22] M. Shaygan, K. Davami, N. Kheirabi et al., "Single-crystalline CdTe nanowire field effect transistors as nanowire-based photodetector," Physical Chemistry Chemical Physics, vol. 16, no. 41, pp. 22687-22693, 2014.

[23] S. Biswas, C. O’Regan, N. Petkov, M. A. Morris, and J. D. Holmes, "Manipulating the growth kinetics of vapor-liquidsolid propagated Ge nanowires," Nano Letters, vol. 13, no. 9, pp. 4044-4052, 2013.

[24] E. I. Givargizov, "Fundamental aspects of VLS growth," Journal of Crystal Growth, vol. 31, pp. 20-30, 1975.

[25] H. Kirmse, W. Neumann, S. Kret et al., "TEM characterization of VLS-grown ZnTe nanowires," Physica Status Solidi (C), vol. 5, no. 12, pp. 3780-3784, 2008.

[26] P. Caroff, K. A. Dick, J. Johansson, M. E. Messing, K. Deppert, and L. Samuelson, "Controlled polytypic and twin-plane superlattices in III-V nanowires," Nature Nanotechnology, vol. 4, no. 1, pp. 50-55, 2009.

[27] Y. F. Chan, X. F. Duan, S. K. Chan, I. K. Sou, X. X. Zhang, and N. Wang, "ZnSe nanowires epitaxially grown on $\mathrm{GaP}(111)$ substrates by molecular-beam epitaxy," Applied Physics Letters, vol. 83, no. 13, pp. 2665-2667, 2003.

[28] X. T. Zhang, T. K. M. Ip, Z. Liu, Y. P. Leung, Q. Li, and S. K. Hark, "Structure and photoluminescence of $\mathrm{ZnSe}$ nanoribbons grown by metal organic chemical vapor deposition," Applied Physics Letters, vol. 84, no. 14, pp. 2641-2643, 2004.

[29] Y. Q. Wang, U. Philipose, T. Xu, H. E. Ruda, and K. L. Kavanagh, "Twinning modulation in ZnSe nanowires," Semiconductor Science and Technology, vol. 22, article 175, 2007. 

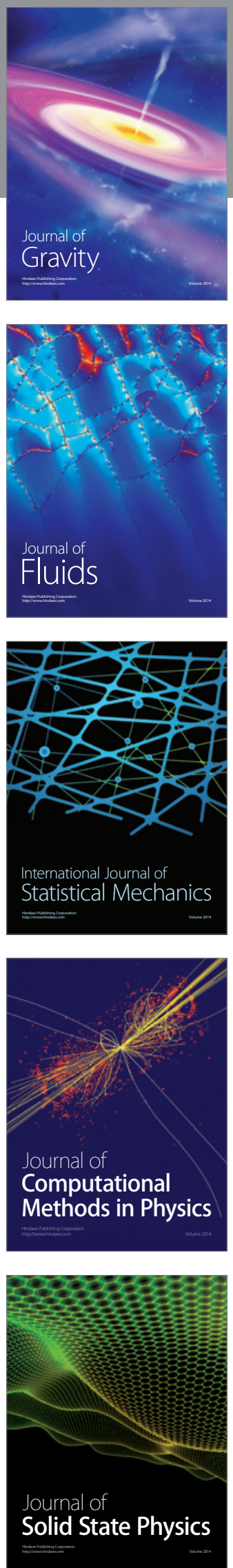

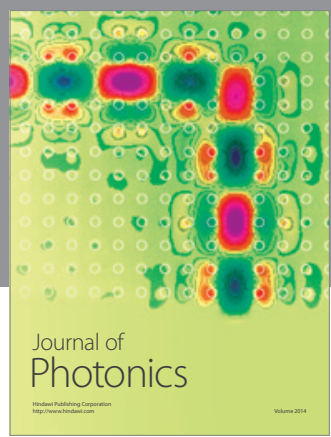

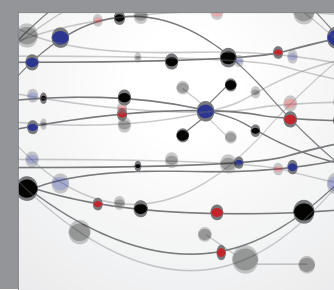

The Scientific World Journal

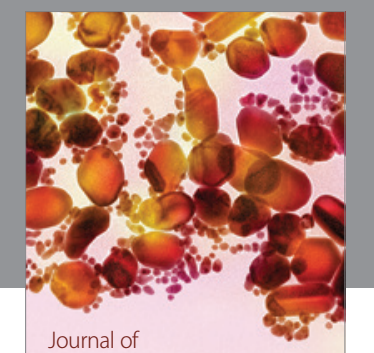

Soft Matter
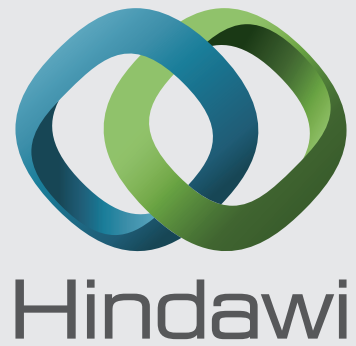

Submit your manuscripts at

http://www.hindawi.com
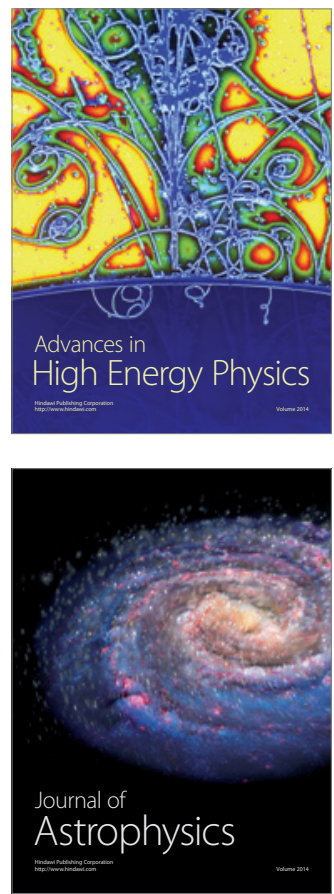
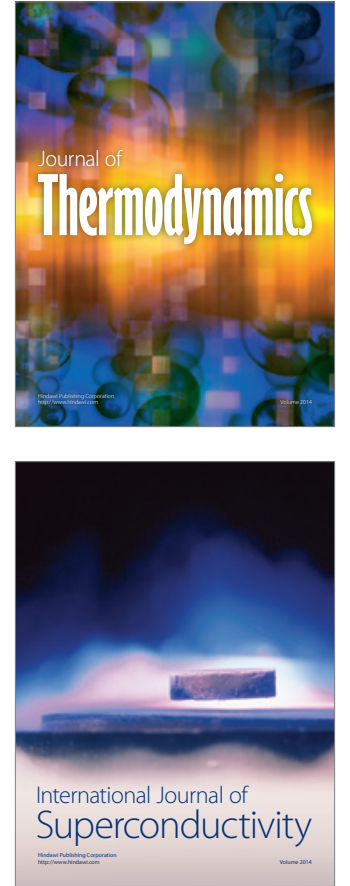
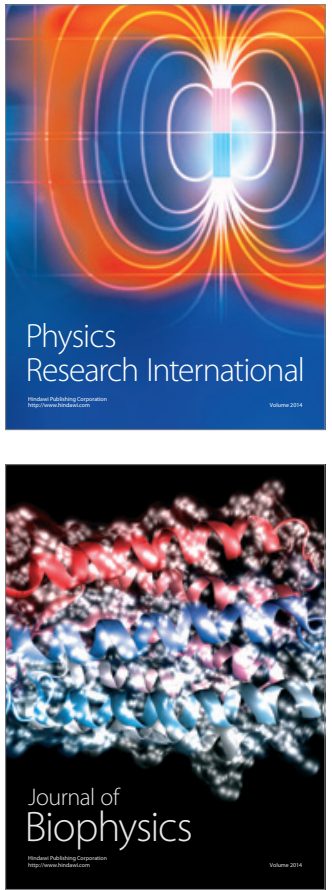
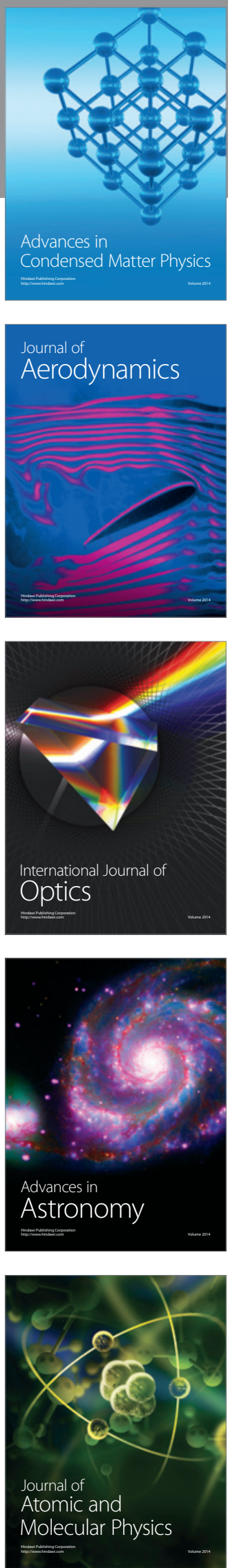